UDK 821.133.1-21.09Corneille P.

\title{
L'ASCENDANT DU DOUTE HYPERBOLIQUE SUR LE PROTOPROLOGUE DES GRANDES TRAGEDIES CORNELIENNES
}

\author{
Boštjan Marko Turk
}

\begin{abstract}
Synopsis
L'étude présente se propose comme objectif de mettre en relief la fonction du protoprologue qui, en présence de son aspect d'archétype, prend le sens du bloc de construction architecturalement principal dans les drames majeurs de cet auteur. On trouve l'élément du protoprologue dans l'exposition des unités les plus importantes de l'opus de Corneille : Il détermine de façon essentielle l'entière pulsation de ce genre de théâtre, qui, par un chaos apparent, s'équilibre en une perspective rationnelle et cartésienne, typique du rationalisme du 17 ème et des siècles suivants. Son facteur déterminant essentiel est le doute hyperbolique, qui ne se suffit pas à lui-même, mais devient l'outil méthodologique de la rationalisation des passions dévastatrices humaines, le tout se déroulant dans le domaine dans lequel la physiologie, au seuil de la nouvelle ère, vient à peine d'entrer, de toucher la frontière ou le contact entre le spirituel et l'organique.
\end{abstract}

Mots-clefs: Pierre Corneille, René Descartes, Rome, folie, rationalisme, protoprologue, passion, tragédie, physiologie, volonté, mort.

Les œuvres cardinales de Pierre Corneille ont toujours été reconnues par la critique comme la voix rationnelle de l'héritage romain, pour lequel l'approche analytique du monde, prévue pour le maîtriser, constitue l'élément sans lequel il n'y a pas de réelle percée dans aucun de ses moments dramatiques. La passion qui tourne à la folie est d'ailleurs fréquemment l'élément du tragique surtout dans la période du classicisme mature de Corneille. Mais l'auteur évite cet élément dès le début, dans le protopro$\operatorname{logue}^{1}$ qui, en présence de son aspect d'archétype, prend le sens du bloc de construction architecturalement principal dans ses drames majeurs. On trouve l'élément du protoprologue dans l'exposition des unités les plus importantes de l'opus de Corneille : Il détermine de façon essentielle l'entière pulsation de ce genre de théâtre, qui, par un chaos apparent (et une folie), s'équilibre en une perspective rationnelle et cartésienne,

${ }^{1}$ L'expression »protoprologue « vient de la théorie dramatique d'Aristote. Il suit le théâtre depuis ses débuts et en ce sens il en est lui-même son »protoélément«. Comparez : "The first scene of all, which preceded the entrance of the chorus, was called the protoprologue and its invention is ascribed to Thespis « (Haigh, A.E. The Tragic Drama of the Greeks. New York: Dover, 1968: 350). 
typique du rationalisme du 17ème et des siècles suivants. Son facteur déterminant essentiel est le doute hyperbolique, qui ne se suffit pas à lui-même, mais devient l'outil méthodologique de la rationalisation des passions dévastatrices humaines, le tout se déroulant dans le domaine dans lequel la physiologie, au seuil de la nouvelle ère, vient à peine d'entrer, de toucher la frontière ou le contact entre le spirituel et l'organique, c'est à dire la matière physique. De façon inductive, l'Histoire de la folie à l'âge classique ${ }^{2}$, qui n'est autre que l'analyse des aberrations humaines en Occident, nous apprend déjà les applications externes de ce genre de conduite. Michel Foucault y donna l'affirmation que la folie, en tant que telle, est en fait, une invention de notre époque contemporaine, et avant tout du cartésianisme, qui, en tant que passion reléguée au dernier rang, l'a éliminée de tout type de discours socialo-constitutif. Foucault a d'ailleurs reconnu qu'avec le rationalisme du 17ème siècle français, le pays a commencé à mettre en place des systèmes d'hôpitaux, dans lesquels on isolait les déséquilibrés, comme les autres institutions de sous-cultures les connaissaient jusque là : des bordels aux bandes de mendiants en passant par les hospices pour indigents. En même temps, il enleva à la folie sont sens généralisant : Il est vrai que le pays ne s'en est rendu compte qu'au seuil de l'ère moderne et l'a ainsi sanctionnée en tant que telle : mais comme chaque époque lui ajoute son interprétation et son sens il en est de même pour elle que pour le doute cartésien : ce n'est que lorsque l'objet du doute deviendra l'objet de mes pensées ${ }^{3}$ que je penserai seulement de la folie qu'elle n'existe pas ou autrement : tant que je douterai d'elle, elle existera, et avec elle je serai moi même normal et non-fou.

La critique moderne dirait que ce n'est qu'avec Foucault qu'il a été possible de penser la folie comme partie intégrante de l'exil social :»Désormais la folie est exilée«, ${ }^{4}$ et c'est aussi la différence, que l'exégèse contemporaine (ainsi que sa raison) établit à travers Descartes entre Sophocle et Corneille. La raison pour laquelle les héros du poète classique maîtrisent aussi bien leurs passions et, à la différence des tragédies grecques, ne cèdent jamais à leur résultante extrême, la folie, est l'époque moderne et plus précisément le cartésianisme. Ce dernier a percé même plus profondément que les stoïques, qui étaient trop rigides aussi bien pour leur époque qu'aujourd'hui. Suréna, le héros éponyme de la tragédie du même nom, ${ }^{5}$ bien que parfait, a une perfection intentionnelle et non empirique : elle est comme un fait ontologique : on peut y croire, on peut s'efforcer de se persuader soi-même et les autres de son existence potentielle, on ne peut pas la sentir, elle échappe à notre expérience et à nos représentations que l'on se fait d'elle. La passion stoïque est un matériel radioactif doré que Descartes transformera par la suite en une matière maîtrisable, qui prouvera la suprématie de l'Homme sur la nature et surtout sur le monde vivant. Mais on ne peut pas se défaire de l'impression qu'il y a déjà beaucoup de „philosophie“ cartésienne dans le chef parthe. On doit cependant à Erich Auerbach, qui se consacra aux analyses de ce sentiment, également en relation avec la folie, dans son œuvre Le Culte des passions, une des meilleures analyses de la passion

\footnotetext{
${ }^{2}$ Foucault, Michel: Histoire de la folie à l'âge classique. Paris: Gallimard, 1961. Comparer aussi: Derrida, Jacques. »Cogito et histoire de la folie». In L'écriture et la différence. Paris: Seuil, 1967.

${ }^{3}$ Dans cet exemple la tautologie n'est pas possible : douter du doute est une constitution de prédicat incorrecte par le sens.

${ }^{4}$ Foucault, Michel. Histoire de la folie à l'âge classique: 82.

${ }^{5}$ Corneille, Pierre. Théâtre, III. Paris: Gallimard, 1960: 413.
} 
${ }^{6}$. Il constatait ainsi que le sage stoïque ne permettra jamais que le monde le domine : c'est que tout contact avec le monde provoquera une contamination : la passion n'est pas seulement contagieuse, mais elle est aussi dangereuse dans son impureté à tous ceux qui se respectent : passio deviendra ainsi le contraire de ratio. „C'est ainsi que passe à l'arrière plan l'opposition initiale entre passio et actio, et que passio devient l'opposé de ratio: aux passions mouvementées s'oppose la quiétude de la raison: mais le mouvement implique une sorte d'activité. Ici, pour la première fois, le mot peut être rendu par notre passion moderne, en partie à cause du mouvement, en partie à cause de l'emportement qu'il suppose toujours pour lest stoïciens: ici se constitue l'image des orages et des remous passionnels, et passio est souvent remplacé par le terme clairement péjoratif de perturbatio «?

Le sentiment difficilement contrôlable opposé à la raison et celui-ci opposé à la folie, dont la passerelle de liaison est justement le dernier concept de la citation d'Auerbach (»perturbatio«), que l'on traduit en „,choc, trouble“ est le domaine Des Passions de l'âme $e^{8}$ de Descartes. Ce débat s'est maintenu dans l'attention également des lecteurs d'aujourd'hui, justement à cause de Corneille, même si ses hypothèses physiologiques et ses théories furent emportées par le temps. On peut lire dans cette œuvre, que la matière cérébrale est liée aux organes sensoriels (les sens), puis aux muscles et au cœur à travers le système nerveux. Celui-ci, le philosophe le comprend comme un ensemble de fins tubules dans lesquels circulent sans cesse des »esprits animaux «, ${ }^{9}$ comme il nomme l'ensemble débridé des souffles, échappements et substances impures qui coulent dans le corps. Notre corps est une machine : son mécanisme de base est la circulation d'une substance jaune, que le cœur chauffe et surtout sa pression est augmentée par les impulsions déclenchées par les sens. Une alerte dans l'environnement extérieur se traduira immédiatement dans tout le corps comme un choc ou une perturbation qui demandera à tout prix une réaction en réponse. Les muscles s'activeront, les articulations se reconnecteront, les membres bougeront, qui seront de ce fait le dernier moteur de l'accomplissement du reflexe de la passion dans le corps. Et enfin, l'être dont la vie et le comportement sont déterminés par les besoins corporels et les instincts, s'enfuira du danger ou bondira hors d'une menace imminente.

Si cette dialectique est entièrement valable pour les animaux, il en est différent pour l'Homme. Celui-ci possède une âme qui est une entité supérieure, liée au corps par une trouvaille sur laquelle on ne peut s'appuyer aujourd'hui. Il s'agit d'une glande particulière glans pinealis (»glande pinéale «), ${ }^{10}$ ou épiphyse aujourd'hui, liée à la sécrétion de la mélatonine, une hormone du sommeil. Intuitivement, Descartes pressentit que la glande pourrait remplir une fonction importante dans l'apaisement (endormissement) de la passion. Le rôle physiologique de cet organe, placé au centre même du cerveau, serait de détecter chaque vapeur de nature animale et de la sublimer.

La glande équipe ensuite l'âme d'une image précise du monde extérieur. La volonté, qui est le mécanisme essentiel des perceptions du classicisme, régule alors

\footnotetext{
${ }^{6}$ Auerbach, Erich. Le Culte des passions. Paris: Macula, 1998.

${ }^{7}$ Auerbach, Erich. Le Culte des passions, p. 56.

${ }^{8}$ Descartes, René. Les Passions de l'âme. Paris: Flammarion, 1996.

${ }^{9}$ Descartes, René. Les Passions de l'âme: 105.

${ }^{10}$ On peut trouver une représentation graphique de la glande ici : http://fr.wikipedia.org/wiki/Glande_pin\% $3 \%$ A9ale.
} 
les „esprits animaux“ dans le sens qu'ils ne représentent plus de danger à la sensation intégrale de dignité du sujet ainsi conçu.

La passion est active et l'Homme est en relation de subordination à elle jusqu'au moment où ses composantes principales atteignent la glande par l'aqueduc de Sylvius, ${ }^{11}$ comme le nomma le philosophe. C'est pourquoi, leur nature est étymologiquement active, patior, l'Homme est le seul être qui, justement, grâce à cet organe, est capable de maîtriser la nature active (et incontrôlable) de la passion. En comparaison avec le personnage stoïque de Suréna, chef parthe et prototype de la tragédie héroïque de Corneille, la passion cartésienne est non seulement isolée et neutralisée, mais elle est en plus mise en rapport avec la raison. Ce n'est que dans cette relation qu'elle devient une des formes de base de la passion, bonne ou mauvaise.

La palette irisée de la passion est soumise à la raison par le fonctionnement de la glande pinéale. Cependant, on peut voir chez les Hommes différents niveaux de réponse à l'action de la passion : certains sont capables de cela avec une force qui fait envie, tandis que pour d'autres cet art reste inconnu, à la fin se retrouvent ceux qui sont entièrement sous le pouvoir de la passion: des personnes maniaques pour lesquelles une réclusion ou un exil dans un établissement psychiatrique est de mise, comme l'enseignait Foucault et comme l'expliqua Descartes, le premier enfant de l'ère moderne tournée vers la maîtrise de la face obscure de l'être humain. L'asile n'est pas tout à fait un concept cartésien, mais c'est une invention entièrement de notre temps. A l'opposé, il y a »l'homme généreux«, un homme généreux ou noble que Les Passions de l'âme définissent de cette manière: »Ainsi je crois que la vraie générosité qui fait qu'un homme s'estime au plus haut point qu'il se peut légitimement estimer, consiste seulement, parti en ce qu'il connaît qu'il n'y a rien qui véritablement lui appartienne que cette libre disposition de ses volontés, ni pourquoi il doive être loué ou blâmé sinon qu'il n'en use bien ou mal, et partie en ce qu'il sent en soi-même une ferme et constante résolution d'en bien user, c'est à dire de ne manquer jamais de volonté pour entreprendre et exécuter toutes les choses qu'il jugera être les meilleures, ce qui est suivre parfaitement la vertu«. ${ }^{12}$

La générosité fait partie des „grandes âmes“ et sert de remède à tout les types d'aberrations que les passions font naître. Ses supports, les nobles généreux, sont déjà par nature (par la structure physiologique de l'épiphyse et par ses implications dans les zones de rangs inférieurs de la matière cérébrale et d'autres composantes du corps) enclins aux grandes choses. Celles-ci détermine le critère : l'essentiel est que l'Homme conserve une relation correcte de la raison envers la passion, de façon à ce qu'elle lui permette toujours d'avoir la »partie gagnante«. Disposer librement de la volonté qui étouffe même la plus bouillonnante des émotions, fait d'eux les géants de leur génération. Rien ne peut les atteindre.

»Je suis maître de moi comme de l'univers;

Je le suis: je veux l'être. O siècles, o mémoire,

Conservez à jamais ma dernière victoire!

Je triomphe aujourd'hui du plus juste courroux

De qui le souvenir puisse aller jusqu'à vous «. ${ }^{13}$

\footnotetext{
${ }^{11} \mathrm{C}$ 'est le canal dorsomédial au dessus du tronc cérébral

${ }^{12}$ Descartes, René. Les Passions de l'ame: 129.

${ }^{13}$ Corneille, Pierre. Théâtre. Paris: Gallimard, 1965: 957.
} 
Ainsi le grand Auguste, une des preuves les plus fortes de la force de la raison et de la volonté sur la passion, décrit la différence entre lui et les conspirateurs, qui menacent sa vie dans un brouillard de passion non libre, sans savoir qu'ils pèchent contre eux-mêmes : à la fin, quand ils se convertissent, ils le reconnaissent

L'Homme de Corneille est grand : sa vocation est la noblesse généreuse, et celle-ci est issue des conséquences pratiques d'une physiologie théorique, qui n'est d'ailleurs utilisable aujourd'hui que comme matériel d'archives. La grandeur des personnages de Corneille, malgré du concept scientifique qui a fait son temps, provient de la maîtrise : Ceci donne une double résultante, la maîtrise de l'Homme en lui-même et comme le dit Auguste „Je suis le maître du monde et je règne sur moi-même“ le monde aussi. C'est seulement grâce à ce mécanisme que l'on peut expliquer pourquoi le fatal protoprologue s'établit toujours de manière à ce que la stature intégrale du héros n'est jamais remise en question. Au contraire, on a plusieurs fois l'impression qu'elle est même renforcée parfois malgré la mort. Dans ce sens on peut suivre un intéressant gradatio in maius qui suit la ligne Auguste - Pompée - Suréna.

Mais les leviers physiologiques seuls ne seraient pas suffisants pour aligner complètement les géants avec la ligne idéale de la générosité noble. Une lecture approfondie nous dévoile donc que la thèse de l'influence de l'épiphyse sur le fonctionnement de l'Homme n'est qu'une formulation plus maladroite des autres idées cartésiennes, qui se sont toutes transférées dans les drames cornéliens et surtout dans la mentalité de l'ère moderne.

Au cœur du rapport entre les sources animales et spirituelles, qui se régule par l'intermédiaire de la fameuse glande, se trouve une connaissance du dualisme, de la dualité. La dualité est établie selon le principe de proportionnalité et ne permet pas d'apaisement. Cette phrase pourrait servir également de synopsis pour expliquer l'essentiel de la matière de l'opus de Corneille. On peut comprendre l'essence du système méthodologique de Descartes comme une exclusion de tous les facteurs qui ne sont pas reliés à la raison et de ce fait invérifiables de la façon gnoséologique la plus exclusive, c'est à dire le doute. C'est justement le doute cartésien qui est la base du cogito, or celui-ci n'est possible qu'à partir du moment où le sujet pensant rejette toutes les impulsions cognitives comme éventuellement inspirées d'un esprit malin : au terme d'une réduction aussi complète, il lui reste le moi pensant, support de l'opération et son produit : le doute. »Il n'y a donc point de doute que je suis, s'il me trompe; et qu'il me trompe tant qu'il voudra, il ne saurait jamais faire que je ne sois rien, tant que je penserai être quelque chose. De sorte qu'après y avoir bien pensé, et avoir soigneusement examiné toutes choses, enfin il faut conclure, et tenir pour constant que cette proposition: Je suis, j'existe, est nécessairement vraie, toutes les fois que je la prononce ou que je la conçois en mon esprit «. ${ }^{14}$ Le sujet pensant est alors devenu, avec l'action de penser (le doute étant l'opération de l'esprit), la dernière base de la réalité, la première autorité de la connaissance au delà de laquelle il n'y a plus rien.

${ }^{14}$ Descartes, René. Méditations métaphysiques. Paris: Flammarion, 2011: 73. Descartes à écrit à ce sujet plusieurs fois : au coeur de la guerre de trente ans, le 10. novembre 1619, auprès d'un poêle chaud quelque part au centre de $1^{\prime}$ Allemagne, à moitié assoupi, un tentateur lui apparait et le persuade que tout ce qu'il vit est une illusion : c'est là que naquit sa méthode qui ventilera le système de pensée occidental. 
C'est pour ainsi dire l'homonyme du drame auquel l'analyse présente se consacre. L'Homme n'est pas seulement grand parce que des glandes le protègent d'un dérapage dans la bestialité : sa grandeur est le produit d'un soupèsement, mais avant tout du doute hyperbolique de solutions potentielles et de la situation donnée. Il est caractéristique pour celle-ci qu'elle soit tout sauf simple et facile. Au contraire, elle est tellement détournée que les critiques ont plusieurs fois reproché au poète l'invraisemblance ou l'improbabilité de sa matière fabulative, de sorte qu'il a dû finalement se réfugier dans la légende pour répondre à ce problème aux critiques et surtout au postulateur de l'art classique »Le vrai peut quelques fois n'être pas vraisemblable ${ }^{15}$ Les protagonistes de ses tragédies se retrouvent en règle générale dans des situations où ils doivent repousser le doute existentiel au point de rester seul, dans leur pensée : cogito signifiera alors aussi un levier d'Archimède avec lequel ils tourneront, dans la suite, le protoprologue à leur avantage. Le héros cornélien est le sujet pensant du doute expansif en tout à part en lui-même. Il s'établit entièrement justement dans la différence qui s'aligne sur celle que Descartes utilisait pour justifier la pensée de la nouvelle ère et de son support.

Plus la situation est impossible, plus fort dubito résonnera dans les termes des protagonistes ; il formera le cogito de l'histoire dramatique dans sa synthèse philosophique aussi inhabituelle également pour notre époque. Don Rodrigue vengera la gifle que son futur beau père infligea à son père. Il battra celui-ci lors d'un duel pour ensuite plonger dans la question clé de ce qui est le plus important entre l'honneur ou l'amour. Il émettra un doute si puissant envers tout (de même que son élue), que leurs êtres (sociologique, érotique et aussi métaphysique) s'effaceront face à tout, sauf devant le fait que Rodrigue (et Chimène) effectuent une opération de l'esprit avec laquelle ils recentrent l'intégralité de la réalité physique et conceptuelle vers un seul dilemme du sujet pensant concrètement pour ce cas, de même qu'en général : L'honneur passe devant l'amour et avant lui il y a le devoir, le code, lex. Pour les protagonistes de la tragicomédie éponyme $\mathrm{Le}$ Cid, il existera le doute de tout, sauf de la pensée créée par une déterminante de base.

Emilia sera le générateur de toute l'histoire dans Cinna (ou la Clémence d'Auguste). La première information que le lecteur reçoit à son sujet est justement le doute. Dans l'exposé présent nous avons inclus l'intégralité du protoprologue de la plus grande tragédie romaine : nous pourrions inclure encore plus de vers, tellement qu'ils reflètent directement tous le noyau du doute selon la position de l'hérö̈ne. Personne ne pourrait, pour ainsi dire, douter d'avantage de sa famille, son origine, la justesse de son comportement en rapport avec son père, Auguste et son amant, qu'Emilia. Citons propédeutique du doute dans son intégralité :
»Durant quelques moments souffrez que je respire,
Et que je considère, en l'état où je suis,
Et ce que je hasarde, et ce que je poursuis.
Quand je regarde Auguste au milieu de sa gloire,
Et que vous reprochez à ma triste mémoire

${ }^{15}$ Boileau, Nicolas. L'Art poétique. Paris: Garnier, 1961: 172. 
Que par sa propre main mon père massacré

Du trône où je le vois fait le premier degré

Quand vous me présentez cette sanglante image,

La cause de ma haine, et l'effet de sa rage,

Je m'abandonne toute à vos ardents transports,

Et crois, pour une mort, lui devoir mille morts.

Au milieu toutefois d'une fureur si juste,

J'aime encore plus Cinna que je ne hais Auguste,

Et je sens refroidir ce bouillant mouvement

Quand il faut, pour le suivre, exposer mon amant.

Oui, Cinna contre moi même je m'irrite

Quand je songe aux dangers où je te précipite.

Quoique pour me servir tu n'appréhends rien,

Te demander du sang, c'est exposer le tien «. ${ }^{16}$

Le cogito d'Emilia met ainsi tout à l'envers sauf la synthèse d'Auguste. Après la phrase la plus connue de l'auteur dramatique:

»Je suis maître de moi comme de l'univers;

Je le suis: je veux l'être. $O$ siècles, ô mémoire,

Conservez à jamais ma dernière victoire!

Je triomphe aujourd'hui du plus juste courroux « ${ }^{17}$

la synthèse d'Emilia est aussi:

Est-il-perte à ce prix qui ne semble légère?

Et quand son assassin tombe sous notre effort,

Doit-on considérer ce que coûte sa mort? ${ }^{18}$

seulement le produit d'une pensée, qui n'est, à la fin tournée que sur elle-même, en fonction de son propre point de départ épistémologique. Le doute et la raison étouffent tout sentiment et passion même s'ils visent le plus grand défi dans l'univers connu alors, la mort du dirigeant absolu, et sont l'outil le plus grand contre la folie. Cette attitude est le contenu du protoprologue.

Le doute hyperbolique apparait de façon encore plus forte dans la tragédie qui est intégralement consacrée à la relation dont la propriété première est d'exclure la probabilité d'une conclusion sur la certitude de quelque chose.

Il n'y a pas de point de support dans Horace, rien de solide, de définitif, en réalité tout est inversé, de la même manière que cela c'est passé dans l'histoire où Descartes était tenté par l'esprit malin ${ }^{19}$. Corneille a placé cela sur la base logique et délicate, là où il semble que même le doute devient déficient. Le sujet de la tragédie est le conflit entre

\footnotetext{
${ }^{16}$ Corneille, Pierre, Théâtre: 902.

${ }^{17}$ Cité supra.

${ }^{18}$ Corneille, Pierre, Théâtre: 903.

${ }^{19}$ Comparez la note 14 .
} 
Albe et Rome, ce qui signifie le conflit de sang le plus proche, généralisé aussi bien sur des personnes représentatives que sur l'histoire elle-même. C'est qu'Albe était la mère de Rome. Numitor était autrefois son roi mais son frère Amule le renversa du trône. La fille de Numitor était ensuite promise à la vie de vestale : elle ne devait pas se marier ou avoir d'enfants. Mais quand le dieu de la guerre, Mars, tomba amoureux d'elle, elle conçut et donna naissance à des jumeaux : l'un d'eux est invoqué directement par le monologue de Sabine dans le début de la tragédie. L'oncle mura vivante la mère, ${ }^{20}$ mais épargna les enfants comme ils étaient issus de sang divin. Romulus et Remus ont été - selon la légende - abandonnés dans un panier en osier dans le Tibre. Ils échouèrent sur la côte où ils furent nourris au début par une louve, puis par la famille d'un paysan. Ils se vengèrent d'Amule lorsqu'ils devinrent adultes et remirent le grand-père Numitor sur le trône. En même temps, ils constatèrent qu'Alba longa était trop petite pour leurs ambitions, c'est pourquoi ils colonisèrent chacun une des collines de ce qui seront plus tard les sept collines de la ville éternelle. Romulus survécut à Remus, car il le tua après un sacrifice. Il reconnut plus tard que ce qu'il avait fait était une erreur et l'enterra avec dignité. Ce n'est qu'avec ce point de vue que le protoprologue d'Horace devient le prolégomène exhaustif de la philosophie cartésienne, ce qui est d'autant plus évident si l'on prend en compte l'ébauche de l'histoire : l'histoire romaine a commencé avec le sang fraternel versé.

\section{»Je suis Romaine, hélas! Puisque Horace est Romain; \\ J'en ai reçu le titre en recevant sa main; \\ Mais ce noud me tiendrai en esclave enchaînée \\ S'il m'empêchait de voir en quels lieux je sois née. \\ Albe, où j'ai commencé de respirer le jour, \\ Albe, mon cher pays, et mon premier amour, \\ Lorsqu'entre nous et toi je vois la guerre ouverte, \\ Je crains notre victoire tant que notre perte. \\ Rome, si tu te plains que c'est là te trahir, \\ Fais-toi des ennemis que je puisse haïr. \\ Quand je vois de tes murs leur armée et la nôtre, \\ Mes trois frères dans l'une et mon mari dans l'autre, \\ Puis-je former des voux et sans impiété \\ Importuner le ciel pour ta félicité «. ${ }^{21}$}

Et cet effusion de sang continue dans l'opposition du doute raisonné contre la base de passion et de folie, que l'on pourrait nommer pour le besoins présent le sexus incestueux, si l'épithète, ne signifie pas pour nous l'inceste, mais une action encore plus futile, celle de verser le sang de son parent. Sabine, à cause de son nom, implique la première action du fondateur de Rome, lorsqu'il lui vînt à manquer de matériel pour la »reproduction«. A l'occasion de la fête de Neptune, »consualia«, il invita dans la ville les habitants des lieux voisins, dont également les Sabines avec les femmes et les filles.

\footnotetext{
${ }^{20}$ Cet exemple semble être une allusion à Antigone de Sophocle et son destin : c'est une sorte de reflet selon le principe antique eros thanatos : 1'une est murée car elle célèbre la mort, et l'autre parce qu'elle apporte la vie.

${ }^{21}$ Corneille, Pierre, Théâtre: 838.
} 
Lorsque le rituel religieux détournait leur attention, les soldats de Romulus s'accaparèrent des femmes et les enlevèrent. ${ }^{22} \mathrm{C}^{\prime}$ est qu'à cette époque, Rome manquait justement de membres du sexe faible et la ville ne pouvait pas grandir au delà de ses frontières.

Sabine, des alexandrins cités ci-dessus, est comme si elle revivait de façon éponyme le destin de ses demi-sœurs du 8ème siècle avant le Christ. »L'enlevée « de la ville de Romulus, comme elle le supplie elle-même, est devenue l'élément constitutif ${ }^{23}$ du nouveau royaume, tout comme jadis ses sœurs dans le désespoir. Ceci déjà est la première impulsion du doute : le second est le pressentiment d'une guerre fratricide qui a lié Romulus et Remus en un destin commun. La vie d'un des frères jumeaux finit sous la pelle assénée de la main même de son frère sur sa nuque. L'ensemble exprime le vers »Tu oses transpercer le cœur de la mère«, ce qui est gradatio in maius distinct et la grande trouvaille de Sabine. En dehors de l'enlèvement, du viol, de l'inceste, du fratricide et du meurtre de son oncle ${ }^{24}$ c'est maintenant le tour de la forme la plus grave de parricide, c'est-à-dire l'assassinat de la mère. Comment un homme raisonné pourrait, face à cela, agir différemment que le philosophe, lors de cette nuit au centre de l'Allemagne, lorsque tout lui semblait si vide de sens que le doute en toute chose était le seul chemin vers la lumière ? Et ceci est l'état émotionnel de base de Sabine dans le protoprologue d'Horace. Même la foi en le plus sacré, en Dieu et les divinités ne l'arrête pas, comme en témoignent ces trois vers :

„Bien loin de m'opposer à cette noble ardeur,
Qui suit l'arrêt des Dieux et court à ta grandeur,
Je voudrais déjà voir tes troupes couronnées,
D’un pas victorieux franchir les Pyrénées,
Va jusqu'en l'Orient pousser tes bataillons;
Va sur le bord de Rhin planter tes pavillons;
Fais trembler sous tes pas les colonnes d'Hercule«. ${ }^{25}$

Ceux-ci en sont la contradiction puisque l'on ne peut pas remplir les commandements des dieux avec une désobéissance diamétrale : c'est que Sabine appelle Rome à partir »vers l'Est « : alors que les divinités l'envoyèrent dans la direction opposée. Il s'agit d'un doute hyperbolique intégral, où tout est flou sauf le cogito.

La continuation du drame aussi signifie l'expérience du doute radical : la figure emblématique et la copie directe de la position intellectuelle de Sabine est sa belle sœur, Camille. Celle-ci effectue seulement un pas de plus de la pensée théorique à la réalisation pratique du doute : au prix de sa vie, elle rejette le résultat de la bataille et le triomphe romain : si elle reconnaissait une telle réalité, elle se trahirait elle-même et le cogito qui la place, en tant qu'être pensant, au dessus de l'ordre contingent des choses dans le monde.

C'est comme si tout ce qui suit ensuite dans la tragédie, confirmerait la connaissance cartésienne. Les forces qui tirent dans le sens contraire sont d'ailleurs tellement en désaccord avec la première phrase du Discours de la méthode de Descartes: »Le bon

\footnotetext{
${ }^{22}$ Ils tuèrent principalement les hommes.

${ }^{23} \mathrm{C}$ 'est le materiel de reproduction.

${ }^{24} \mathrm{~L}$ 'expression latine est avunculicidus - Les jumeaux tuent le frère de leur père, Amule.

${ }^{25}$ Corneille, Pierre, Théâtre: 838.
} 
sens est la chose du monde la mieux partagée «, ${ }^{26}$ que Corneille a dû baser la plausibilité de son histoire non pas sur une réalité de vie mais sur l'authenticité de la légende de Livie. Peut-on attendre que le père (le vieux Horace), lorsqu'il apprend que son fils est le dernier survivant de la bataille, maudirait celui-ci pour couardise ? Est-il possible qu'ensuite le jeune Horace, survivant miraculé, vaincrait les Curiaces absolument seul et tuerait sa sœur ? Est-ce que c'est ensuite plausible que le vieil Horace fêterait cette fois-ci ses actions ? Et ensuite : qu'il obtiendrait pour lui une grâce de l'empereur Tulle? Et finalement, que ce dernier comprendrait tout cela et conclurait de façon heureuse cette histoire totalement malheureuse ?

Ainsi on arrive à la conclusion qui accompagne les tragédies de Corneille. La sortie du protoprologue n'a qu'un sens secondaire en comparaison avec le dilemme de base que ce dernier établit. ${ }^{27}$ La mort de Pompée est l'exemple gradué du même genre. Au tout début, on trouve le doute hyperbolique. Photin, Achillas, Ptolomée et Septime débattent de toutes leurs forces sur l'attitude à adopter. Ne rien faire ? Cacher le chef militaire ? Le sauver? Le livrer à César? Le tuer?

»C'est de quoi, mes amis, nous avons à résoudre.

Il apporte en ces lieux les palmes ou la foudre:

S'il couronna le père, il hasarde le fils;

Et nous l'ayant donnée, il expose Memphis.

Il faut le recevoir, ou hâter son supplice,

Le suivre ou le pousser dedans le précipice.

L'un me semble peu sûr, l'autre peu généreux,

Et je crains d'être injuste, et d'être malheureux.

Quoi que je fasse enfin, la fortune ennemie

M'offre bien des périls, ou beaucoup d'infamie:

C'est à moi de choisir, c'est à vous d'aviser

A quel choix vos conseils doivent me disposer.

Il s'agit de Pompée, et nous aurons la gloire

D'achever de César ou troubler la victoire:

Et je puis dire enfin que jamais potentat

N'eut à délibérer d'un si grand coup d'État«.28

Finalement, ils décident de faire un attentat: les avis sont que Cléopâtre se rapproche trop de César pour pouvoir laisser Pompée en vie. De ce fait, ils le tuent. Toute

${ }^{26}$ Descartes, René. Discours de la méthode. Paris: Flammarion, 1996: 33.

${ }^{27} \mathrm{Le}$ Cid, que nous n'avons cité qu'en introduction, est le jumeau dramaturgique d'Horace. Basé sur le doute radical des personnes dans des situations identiquement impossibles comme le sont Camille et Sabine. A la fin, la solution est aussi provisoire : Le deus ex machina apporte le dénouement voulu qui tourne la tragédie en tragi-comédie. S'il y a des drames qui se ressemblent vraiment, ce sont ces deux là. Chimene et Rodrigue doutent et réfléchissent sans cesse sur la possibilité qui est la plus adaptée pour le futur. Sur la base de cette »vivisection « d'alternatives se forme leur identité, se confirme leur sentiment mutuel et renforce la conscience d'autonomie du sujet. Bien que Le Cid mérite une analyse au début du texte, on ne désigne le problème que dans la partie marginale. Que l'étude sur le phénomène de Jean Racine, écrit par Roland Barthes, serve d'excuse (Barthes, Roland. Sur Racine Paris: Seuil, 1963). De nombreuses affirmations de base sur la structure psycho-historique des drames de Racine y sont traitées sous forme de notes.

${ }^{28}$ Corneille, Pierre, Théâtre: 1050. 
l'action sera ensuite tournée en arrière par César : Il le retournera littéralement au protoprologue et lavera l'honneur du chef assassiné. Le premier vers nous dirige littéralement dans ce sens, comme suit : »Il est juste, et César est tout prêt de vous rendre / ce reste où vous avez tant de droit de prétendre $\ll^{29}$

César remet ainsi les choses du monde dans leur état initial : Ceci est l'une des manières qu'on a déjà vue dans Le Cid, Polyeucte et Horace : c'est la méthode complémentaire pour faire revenir les choses à leur point de départ intégral. Ici, il possède aussi cette propriété supplémentaire montrant qu'il s'agit d'un double doute. La plus haute autorité du monde de l'époque place ce que pense Ptolémée et ses conspirateurs dans le point de départ radical de négation (donc le doute). Comme si le drame n'avait jamais eu lieu.

Le rationalisme, basé sur le scepticisme d'un côté et sur la représentation de la »noblesse généreuse « de l'autre, n'est pas visible uniquement dans les postulats physiologiques, les questions sur le dualisme philosophique : res cogitans versus res extensis ou la chose pensante (cogito) en opposition à la matière animale, et ensuite dans le doute méthodique qui mène le sujet pensant, mais représente l'appareil d'expression de la totalité des figures de Corneille, du moins de celles pour lesquelles s'applique que l'hypophyse étouffe l'impulsivité impure et les oriente vers l'idéal de la grandeur parfaite. ${ }^{30} \mathrm{Il}$ est aussi reconnaissable en ce qui concerne les quatre règles (la procédure planifiée). Une fois que le doute est arrivé au tabulae rasae de ce qu'il a dû supprimer comme étant un fantasme de la connaissance, le cogito a orienté la méthodologie de sa connaissance vers le chemin de la rationalisation des objets de connaissance. Il est arrivé ici au point clé qu'il a défini dans le sens que la pensée sans objet ne peut exister : L'Homme pensant s'établit donc dans le doute et ce dernier dans l'objet de la pensée : »Nulle pensée sans objet «. ${ }^{31}$ La règle de l'analyse est aussi la règle première des drames de Corneille, ou du moins de ses protoprologues. Toutes les figures de ce théâtre cherchent un objet sur lequel elles appliqueraient leur stature d'identité. Le mot »objet « qui est aussi important que la relation relative à la dernière phrase subordonnée, est la composante clé des drames de Corneille. »Digne objet de ma gloire « est la phrase qui résonne un nombre incalculable de fois dans ses drames. Le message qu'il apporte est simple à comprendre : le nouveau sujet doit s'établir dans le monde objectif, c'est pourquoi il cherche l'objet de sa réalisation. On pourrait ici de nouveau tout recommencer depuis le début, revenir à Chimène et Rodrigue, doubler cela sur Horace, arriver à la relation entre César et la veuve de Pompée et nous retrouverions toujours la même situation.

Celle-ci pourrait bien présenter l'enjeu dialéctique de ce type du théâtre et mettre en question l'idée du tragique dans l'oeuvre cornéllienne.

\footnotetext{
${ }^{29}$ Corneille, Pierre, Théâtre: 1102.

${ }^{30}$ Ils ne sont pas tous ainsi - En accord avec les convictions de Descartes - : Emilie, Ptolomée, Don Gomes, Felix et les autres. Ceux-ci sont restés au niveau de 1'humanoïde qui est plus proche du monde animal qu'il n'y paraît.

${ }^{31}$ Cette expression est 1'une des formules de Descartes, elle signifie cependant la même chose que ce qu 'indiquent les lignes précédentes.
} 


\section{BIBLIOGRAPHIE :}

Auerbach, Erich. Le Culte des passions. Paris: Macula, 1998.

Barthes, Roland. Sur Racine. Paris: Seuil, 1963.

Boileau, Nicolas. L'Art poétique. Paris: Garnier, 1961.

Corneille, Pierre. Théâtre. Paris: Gallimard, 1965.

Corneille, Pierre. Théâtre, III. Paris: Gallimard, 1960.

Derrida, Jacques. »Cogito et histoire de la folie». In L'écriture et la différence. Paris: Seuil, 1967.

Descartes, René. Discours de la méthode. Paris: Flammarion, 1996.

Descartes, René. Les Passions de l'âme. Paris: Flammarion, 1996.

Descartes, René. Méditations métaphysiques. Paris: Flammarion, 2011.

Foucault, Michel: Histoire de la folie à l'âge classique. Paris: Gallimard, 1961.

Haigh, A.E. The Tragic Drama of the Greeks. New York: Dover, 1968. 